\title{
Schematisation of Tree Drawings
}

\author{
Joachim Gudmundsson ${ }^{1}$, Marc van Kreveld ${ }^{2}$, and Damian Merrick ${ }^{1,3}$ \\ ${ }^{1}$ National ICT Australia*, Sydney, Australia \\ \{joachim.gudmundsson, damian.merrick\}@nicta.com.au \\ ${ }^{2}$ Department of Computer Science, Utrecht University, The Netherlands \\ marc@cs.uu.nl \\ ${ }^{3}$ School of Information Technologies, University of Sydney, Australia
}

\begin{abstract}
Given a tree $T$ spanning a set of points $\mathcal{S}$ in the plane, we study the problem of drawing $T$ using only line segments aligned with a fixed set of directions $\mathcal{C}$. The vertices in the drawing must lie within a given distance $r$ of each original point $p \in \mathcal{S}$, and an objective function counting the number of bends must be minimised. We propose five versions of this problem using different objective functions, and algorithms to solve them. This work has potential applications in geographic map schematisation and metro map layout.
\end{abstract}

\section{Introduction}

A schematic drawing is one in which line segments conform to a restricted set of orientations, often for the purpose of increasing readability. Schematic drawings are widely used in many application areas, including electronics, software engineering and visualising geographical networks.

There has been extensive research into orthogonal graph drawing, where edges of a graph are drawn as sequences of alternating horizontal and vertical line segments; see Eiglsperger et al. [5] for a survey. Lauther and Stübinger [8], and subsequently Brandes et al. [2], investigated the problem of generating orthogonal schematic plans from sketch drawings. Hong et al. [7, Stott and Rodgers [13] and Nöllenburg and Wolff [12] looked at the layout of metro maps, in which lines generally conform to horizontal, vertical and diagonal $\left(45^{\circ}\right.$ and $\left.135^{\circ}\right)$ orientations.

A popular topic in cartography is polygonal line simplification, and this has also been investigated in a setting of restricted orientations [10 11. Cabello et al. 3] presented an algorithm to construct various types of schematisations of geographical networks, in which vertex positions remain unmodified but edges are redrawn in a schematised way.

Particularly of interest are certain point placement problems in computational geometry. Cabello and van Kreveld [4] study the problem of aligning as many points as possible in a given set of orientations. If a planar graph is defined on

* National ICT Australia is funded through the Australian Government's Backing Australia's Ability initiative, in part through the Australian Research Council. 

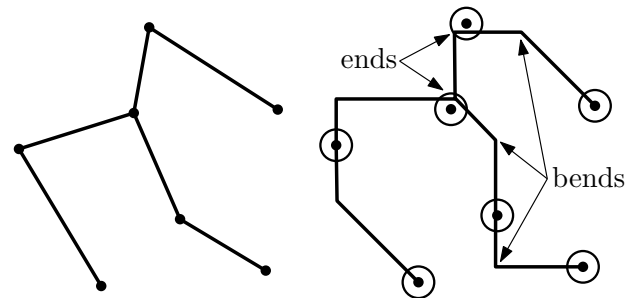

(a)

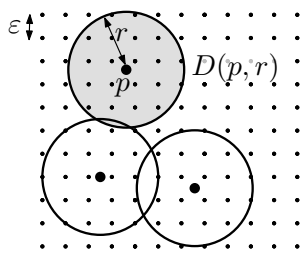

(b)

Fig. 1. (a) A tree (left) and a schematisation of the tree (right). Some bends (along edges) and ends (at vertices) are marked. (b) The grid $G_{\varepsilon}$.

the points, and only points connected in the graph are considered, they prove the problem NP-hard, even when only one orientation is used for alignment. They show that discrete forms of the problem can be solved more efficiently, however. This motivates us to discretise our own problems; we detail this later in this section.

In this paper, we aim to produce schematic drawings of trees from initial sketch, geographical or automatic layouts. We propose five problems. Each takes as input a tree $T$ spanning a point set $\mathcal{S}$, and the output should be a schematisation of the tree's initial layout (See Fig. 1(a)).

To be considered a schematisation, we require that line segments in the drawing align with certain orientations, and that they pass within a certain distance of each original point $p \in \mathcal{S}$. In addition, we want to minimise an objective function $M(p)$ defined for each point $p \in \mathcal{S}$. $M(p)$ measures the complexity of the lines from $p$ to all of $p$ 's children in $T$.

We formally define the concept of a tree schematisation as follows.

Definition 1. Given a tree $T$ spanning a point set $\mathcal{S}$, a set of directions $\mathcal{C}$ and a real number $r>0$, a tree schematisation $T^{\prime}$ of $T$ is a layout of $T$ such that:

1. every line segment of $T^{\prime}$ is aligned with a direction $c \in \mathcal{C}$,

2. for every $p \in S$, the corresponding point $p^{\prime}$ in the schematisation lies inside the disc $D(p, r)$ centred at $p$ with radius $r$

3. $\sum_{p \in \mathcal{S}} M(p)$ is minimised.

The complexity of a tree schematisation can be measured in a number of ways. The primary difference between the five problems we propose lies in the objective function $M(p)$. The choice of $M(p)$ defines how to calculate a cost for each point of a given schematisation. Each problem bases this choice on a particular assumption of how to measure complexity. Some of the problems also take additional input in the form of a set of coloured paths covering $T$, which in turn affects the objective function.

Henceforth, we call the five problems $\pi_{1}-\pi_{5}$. The objective functions associated with each of these are denoted $M_{1}(p)-M_{5}(p)$. We refer to the value of these functions for a schematisation as the $M_{1}$ cost, $M_{2}$ cost, etc. 
Each objective function measures the number of bends and ends in a given schematisation, as illustrated in Fig. 11(a). We use the term bend to mean a change in direction of a line between two vertices in the tree $T$. We use end to mean a bend at a vertex; that is, a line that does not continue through a vertex in a single direction. What counts as an end depends on the particular problem; we detail this in the following sections.

In this paper, we assume that the set of directions $\mathcal{C}$ is constructed by dividing the angle $2 \pi$ by a given even integer $m$ of desired directions, i.e. the $i$ th direction in $\mathcal{C}$ is defined by an angle of $\frac{2 \pi i}{m} . m$ must be even to ensure that for every direction $c \in \mathcal{C}$, the opposite direction $\bar{c}$ is also in $\mathcal{C}$. We look at a discretised version of the general problem of constructing tree schematisations. We place a finite set of points inside each disc $D(p, r)$, and the constructed schematisation must choose one of these points for the position of the vertex $p \in T$. We use a global grid $G_{\varepsilon}$ with $\varepsilon$ horizontal and vertical spacing between grid points to obtain the set of points $G_{\varepsilon}(p)$ for each disc $D(p, r) . G_{\varepsilon}(p)$ is the set of grid points in $G_{\varepsilon}$ that lie inside $D(p, r)$. There are $\mathcal{O}\left(\frac{r^{2}}{\varepsilon^{2}}\right)$ such points (See Fig. 1(b)).

Sections 2 and 3 define $\pi_{1}-\pi_{5}$ in detail. Section 4.1 presents a general method for computing tree schematisations on the grid $G_{\varepsilon}$. This method runs in time exponential in the degree of the tree. In many applications, the degree may be considered constant. In this case, the time complexity of the method is $O\left(\frac{r^{4}}{\varepsilon^{4}}|\mathcal{C}|^{(3+\Delta(T))} n\right)$, where $\Delta(T)$ is degree of $T$ and $n$ is the number of vertices. However, $\pi_{1}-\pi_{5}$ can be solved in time polynomial in the degree of the tree; Sections 4.2 - 4.5 give algorithms for this, based on the general method.

\section{Coloured Tree Problems}

Problems $\pi_{1}$ and $\pi_{2}$ involve coloured trees; they take as input a set $\mathcal{P}$ of elementary paths covering the tree $T$, with each path identified by a distinct colour (See Fig. 2).

Problem $\pi_{1}$ : Single edges only. In $\pi_{1}$, we assume that the paths in the set $\mathcal{P}$ are edge-disjoint (as in Fig. 2(a)), and we want to minimise the number of bends and ends along each of the coloured paths independently.

Choose an arbitrary leaf of $T$ to be the root vertex. For any vertex $p$ in $T$, let $N(p)$ be the set of vertices adjacent to $p$ and let $\operatorname{ch}(p) \subseteq N(p)$ be the set of $p$ 's children. We define $\pi_{1}$ 's objective function $M_{1}(p)$ as:

$$
M_{1}(p)=\sum_{q \in N(p)} \operatorname{ends}_{1}(q, p)+\sum_{q \in \operatorname{ch}(p)} 2 \times \operatorname{bends}(q, p)
$$

where $\operatorname{ends}_{1}(q, p)=0$ if there is an edge of the same colour as (q, p) entering $p$ from the opposite side, or 1 otherwise, and bends $(q, p)$ is the number of bends along the edge between $q$ and $p$. Fig. 3 shows examples. The multiplication of bends by 2 equates the cost of a bend in an edge with two ends at a vertex, which may visually appear the same. 


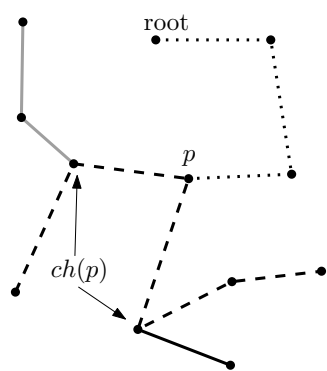

(a)

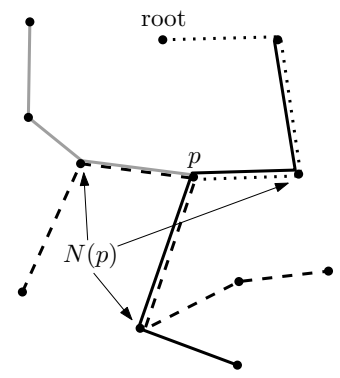

(b)

Fig. 2. An example of a "coloured tree", with solid, dashed, dotted and light grey lines representing four different coloured paths. The left example shows an edge-disjoint path cover $\left(\pi_{1}\right)$, and the right shows a path cover with multiple edges allowed $\left(\pi_{2}\right)$. The children $\operatorname{ch}(p)$ and neighbourhood $N(p)$ of the point $p$ are illustrated.
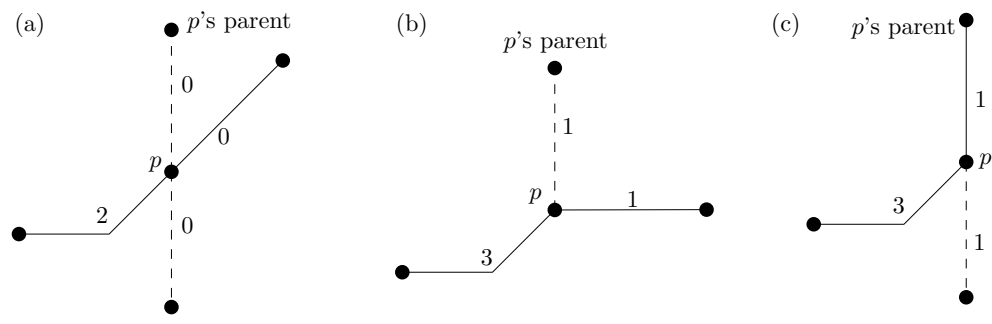

Fig. 3. Counting ends and bends in $\pi_{1}$. A bend costs the same as two ends. The $M_{1}(p)$ costs are $(\mathrm{a})(1$ bend $)=2$, (b) $(1$ bend +3 ends $)=5$, and $(c)(1$ bend +3 ends $)=5$.

Problem $\pi_{2}$ : Multiple independent edges. Problem $\pi_{2}$ removes the requirement for the coloured paths to be edge-disjoint; more than one path may contain an edge between the same two vertices (See Fig. 2(b)). Given multiple edges in the input, it holds that $M_{2}(p)=M_{1}(p)$. Every edge between the same pair of vertices counts towards the cost, where in $\pi_{1}$ there could only be one edge between each adjacent pair of vertices.

$\pi_{2}$ requires that the endpoints of multiple edges between two nodes must coincide in the schematisation, but not the number of bends in the edge nor the orientations of the line segments.

\section{Uncoloured Tree Problems}

The three remaining problems, $\pi_{3}-\pi_{5}$, take uncoloured trees; they assume that no covering set of paths is given. This leads to ambiguity in how to count ends, particularly when many lines in the schematisation enter a vertex in the same direction. $\pi_{3}-\pi_{5}$ model three different possibilities. 
(a)

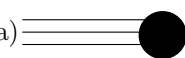

(b)

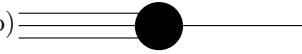

(c)

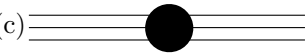

Fig. 4. Three lines entering a vertex in the same direction, with (a) no lines, (b) one line and (c) three lines in the opposite direction

Problem $\pi_{3}$ : Parallel lines remain separate. $\pi_{3}$ assumes that multiple edges, or any lines entering a vertex in a single direction, are drawn separately. For every line entering a vertex in a certain direction, there must be another line entering the vertex in the opposite direction, otherwise the line is counted

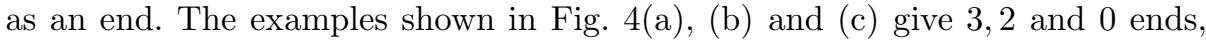
respectively. The objective function is:

$$
M_{3}(p)=\sum_{c \in \mathcal{C}} \frac{1}{2} \times|\operatorname{edges}(p, c)-\operatorname{edges}(p, \bar{c})|+\sum_{q \in \operatorname{ch}(p)} 2 \times \operatorname{bends}(q, p)
$$

where edges $(p, c)$ is the number of $c$-directed lines entering $p$, and edges $(p, \bar{c})$ is the number of lines entering $p$ in the opposite direction to $c$. The factor of $\frac{1}{2}$ ensures counting ends in each pair of opposing directions only once.

Problem $\pi_{4}$ : Parallel lines merge to line on opposite side. Problem $\pi_{4}$ assumes that lines entering a vertex from the same direction are allowed to merge without cost inside the vertex, given the presence of at least one line in the opposing direction. If there is no line in a given direction, an end is counted for every line in the opposing direction. If one or more lines are placed in a pair of opposing directions, there are no ends.

$$
M_{4}(p)=\sum_{q \in N(p)} \operatorname{ends}_{4}(q, p)+\sum_{q \in c h(p)} 2 \times \operatorname{bends}(q, p)
$$

where ends ${ }_{4}(q, p)=0$ if there is any edge entering $p$ from the opposite direction to the edge $(q, p)$, or 1 otherwise. In this problem, the examples in Fig. 4(a), (b) and (c) produce 3, 0 and 0 ends respectively.

Problem $\pi_{5}$ : Parallel lines always merge. The final problem, $\pi_{5}$, assumes that lines entering a vertex from the same direction are drawn as a single line; that is, parallel lines merge to a single line before a vertex. As soon as at least one line is present in the opposite direction, no ends are counted. $M_{5}(p)$ is equal to:

$$
\frac{1}{2} \sum_{c \in \mathcal{C}}|\operatorname{sign}(\operatorname{edges}(p, c))-\operatorname{sign}(\operatorname{edges}(p, \bar{c}))|+2 \sum_{q \in \operatorname{ch}(p)} \operatorname{bends}(q, p)
$$

where $\operatorname{sign}(x)=1$ if $x>0$, or 0 otherwise. For the examples in Fig. [t the number of ends are 1,0 and 0 for (a), (b) and (c) respectively.

\section{Algorithms for Tree Schematisation}

In this section, we present an algorithm to produce a schematisation of a given tree, without considering the specific objective function being used. The algorithm is then modified for each of the problems $\pi_{1}-\pi_{5}$ to provide a more efficient 
solution in each case. In all cases, we use the grid discretisation introduced in Section 1. First, we define an important concept used throughout this section.

Definition 2. Given a set of directions $\mathcal{C}$, a $\mathcal{C}$-directed path between two points $p$ and $q$ is a polygonal chain from $p$ to $q$ in which every line segment is parallel to some direction in $\mathcal{C}$.

\subsection{A General Solution}

Start by choosing an arbitrary leaf vertex to be the root vertex $p_{\text {root }}$ of the tree $T$. Perform a post-order traversal on $T$, visiting each point $p \in \mathcal{S}$ once from the leaves to the root. The algorithm finds optimal solutions for each subtree $T_{p}$ of $T$ rooted at $p$, and during its traversal of $T$ propagates these solutions upward, until a complete solution has been generated at the root vertex. We use the term $M$ cost to denote the value of the objective function $M(p)$. The $M$ cost of a subtree of $T$ is the summed $M$ cost of all points in the schematisation of the subtree.

For each vertex $p$, given a grid point $p^{\prime} \in G_{\varepsilon}(p)$ and an direction $c \in \mathcal{C}$, let $M_{\text {min }}\left(p, p^{\prime}, c\right)$ be the minimum $M$ cost of the subtree of $T$ rooted at $p$, assuming that the schematisation passes through $p^{\prime}$, and that the path from $p$ to $p$ 's parent will leave $p^{\prime}$ in direction $c$. We will refer to the direction $c$ as the parent direction from $p$. We assume in this description that there is only one edge from $p$ to $p$ 's parent, and hence only one $\mathcal{C}$-directed path can be constructed in the schematisation. In the problem $\pi_{2}, p$ may have multiple edges to its parent, and the schematisation may use different directions for each. In this case, we perform the same steps several times, once for each edge. This process is further detailed in Section 4.2 .

When the algorithm visits the vertex $p$, it computes and stores for every point $p^{\prime} \in G_{\varepsilon}(p)$, and for every parent direction $c \in \mathcal{C}$ the value of $M_{\min }\left(p, p^{\prime}, c\right)$, along with the corresponding solution. If $p$ has $k$ children, then $k$ paths must be stored for each solution. Thus $\mathcal{O}\left(\frac{|\mathcal{C}| k r^{2}}{\varepsilon^{2}}\right)$ values are stored at each vertex $p$.

As introduced in Section [1 the objective function being minimised by the algorithm may be divided into a count of bends and ends. We can count the number of bends by considering each child $q \in \operatorname{ch}(p)$ in turn.

Given two grid points $p^{\prime} \in G_{\varepsilon}(p), q^{\prime} \in G_{\varepsilon}(q)$, let $\delta\left(q^{\prime}, p^{\prime}, c_{1}, c_{2}\right)$ be the minimum number of bends needed by a $\mathcal{C}$-directed path from $q^{\prime}$ to $p^{\prime}$ that starts in direction $c_{1} \in \mathcal{C}$ and ends in direction $c_{2} \in \mathcal{C}$. This value can computed in $O(1)$ time. Compute $\delta\left(q^{\prime}, p^{\prime}, c_{1}, c_{2}\right)$ for every pair of grid points $p^{\prime} \in G_{\varepsilon}(p), q^{\prime} \in G_{\varepsilon}(q)$ and for each pair of directions $c_{1}, c_{2} \in \mathcal{C}$. These values are the minimum number of bends required for any possible path from $q$ to $p$. Now add $M_{\min }\left(q, q^{\prime}, c_{1}\right)$ to these and store the minimum solution for every child $q$, grid point $p^{\prime}$, final path direction $c_{2}$, and parent direction $c$. To calculate $M_{m i n}\left(p, p^{\prime}, c\right)$, it remains only to count the number of ends.

Unlike bends, ends cannot generally be counted independently for each of $p$ 's children. Let $k$ be the number of children of $p$. If $k \leq 1$, i.e. $p$ is a leaf vertex or a vertex with only one child, then only the edge between $p$ and $p$ 's parent, or its 
interaction with the single child edge, needs to be considered in calculating the number of ends.

If $k>1$ then the solution will, in general, be far more complicated, since the end cost of the $\mathcal{C}$-directed path from each child will depend on the paths from the other children. It can be shown that it is NP-hard to compute the solution with minimum end cost, even in a somewhat restricted case (See Section 4.5).

A brute force approach can be used to explore all solutions over the entire set of $p$ 's children. There are $\left(\begin{array}{c}|\mathcal{C}| k \\ k\end{array}\right)$ such solutions, which is a number exponential in $k$. Sections 4.2 - 4.5 give methods to compute minimum end cost solutions for $\pi_{1}-\pi_{5}$ that need only time polynomial in $k$.

Once $p_{\text {root }}$ has been processed, the entire tree has been traversed. Now find the minimum value of $M_{\text {min }}\left(p_{\text {root }}, p^{\prime}, c\right)$ over all grid points in $G_{\varepsilon}\left(p_{\text {root }}\right)$. A schematisation can be traced back through the tree, from root to leaves, following stored solutions of minimum cost at each vertex.

Since we start by finding a locally optimal solution at each leaf vertex, and then augment these solutions to find an optimal solution for the subtree rooted at every parent vertex, it is clear by induction that the solution obtained at $p_{\text {root }}$ is optimal for the entire tree.

Time complexity. The time complexity of the algorithm depends on the specific problem being solved. However, a minimum time complexity of $\Omega\left(\frac{r^{4}}{\varepsilon^{4}}|\mathcal{C}|^{3} k\right)$ for processing each vertex $p$ can be noted, as in all five cases $\delta\left(q^{\prime}, p^{\prime}, c_{1}, c_{2}\right)$ values must be computed for the $k$ children of $p$. This equates to $\Omega\left(\frac{r^{4}}{\varepsilon^{4}}|\mathcal{C}|^{3} n\right)$ over an entire tree of $n$ vertices.

\subsection{Problems $\pi_{1}$ and $\pi_{2}$}

The objective function $M_{1}(p)$ counts bends and ends only along each individual coloured path. Hence, the solution for one path has no effect on the solution for another, and they may be treated independently. $M_{2}(p)$ is the same, but multiple edges must be taken into account.

The algorithm. For any coloured path containing the vertex $p$, one can consider all solutions in polynomial time, since there are at most two edges of that colour incident to $p$. Find the best solution for every colour, and sum the $M_{1}$ costs to get $M_{\min }\left(p, p^{\prime}, c\right)$ over all of $p$ 's children.

In $\pi_{2}$, there may be multiple coloured edges from $p$ to $p$ 's parent. In this case, consider a different parent direction for each colour in turn, and repeat the above process. As the colours are independent, choose the best solution for each and sum them to get the total $M_{2}$ cost.

Time complexity. We compute the $M_{\min }\left(p, p^{\prime}, c\right)$ values for each $p \in \mathcal{S}$ in time $\mathcal{O}\left(\frac{r^{4}}{\varepsilon^{4}}|\mathcal{C}|^{3} k\right)$. We visit each of the $n$ points in the tree only a constant number of times, so a total of $\mathcal{O}\left(\frac{r^{4}}{\varepsilon^{4}}|\mathcal{C}|^{3} n\right)$ time is needed to solve $\pi_{1}$. The time complexity for $\pi_{2}$ increases in the worst case by a factor of $|\mathcal{P}|$, giving $\mathcal{O}\left(\frac{r^{4}}{\varepsilon^{4}}|\mathcal{C}|^{3}|\mathcal{P}| n\right)$ overall. 


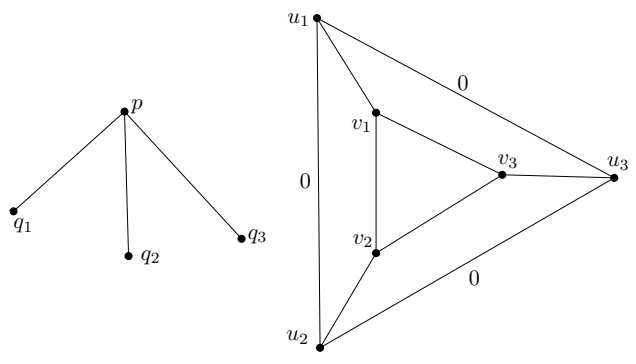

(a)

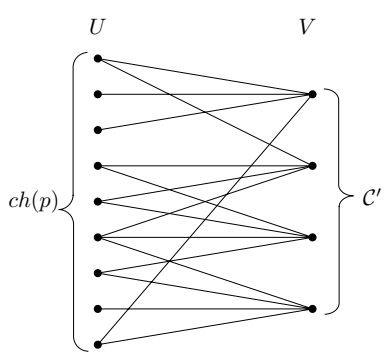

(b)

Fig. 5. (a) A vertex $p$ with three neighbours $q_{1}, q_{2}, q_{3}$ (left) and the corresponding matching graph $G=(V, E)$ (right). (b) The bipartite matching graph $G=(U, V, E)$ constructed for $\pi_{4}$. Every node in $V$ is connected by $|V|=\left|\mathcal{C}^{\prime}\right|$ edges to nodes in $U$, representing the $\left|\mathcal{C}^{\prime}\right|$ lowest cost paths entering $p$ in the corresponding direction.

\subsection{Problem $\pi_{3}$}

In order to minimise the cost function $M_{3}(p)$, we want to match each individual $\mathcal{C}$-directed path entering $p$ with a different path coming in to $p$ in the opposite direction. We only want to do this if the number of bends on the edges does not overcome the reduced end cost, however.

We propose a transformation from this problem to a minimum cost maximum cardinality matching problem. A matching on a graph is a set of vertex-disjoint edges. A maximum cardinality matching is such a set that is as large as possible. A minimum cost maximum cardinality matching is a maximum cardinality matching in which the sum of edge weights is minimal. A detailed introduction to graph matching can be found in the literature [9]. Gabow [6] details an algorithm to solve weighted matching problems for a graph $G=(V, E)$ in time $\mathcal{O}(|V|(|E|+|V| \log |V|))$.

The algorithm. Let $q_{1}, q_{2}, \ldots, q_{|N(p)|}$ be the neighbouring vertices of $p$ in $T$. Consider in turn each $q_{i}, 1 \leq i \leq|N(p)|$. For every direction $c_{2} \in \mathcal{C}$, compute the minimum $M_{3}$ cost assuming that the minimum-bend $\mathcal{C}$-directed path from $q_{i}$ to $p$ enters $p$ in direction $c_{2}$ and that there is no matching path in the direction opposite to $c_{2}$. Store the minimum of these values, and the associated direction. Consider now every pair of $p$ 's neighbours $q_{i}, q_{j}, 1 \leq i \leq|N(p)|, 1 \leq j \leq|N(p)|$, and similarly compute and store the minimum $M_{3}$ cost solution for every $c_{2} \in \mathcal{C}$ assuming that the $\mathcal{C}$-directed path from $q_{i}$ enters $p$ in direction $c_{2}$ and the path from $q_{j}$ enters in the direction opposite to $c_{2}$. Ties for the minimum cost may be broken arbitrarily. If either $q_{i}$ or $q_{j}$ is $p$ 's parent, consider only the parent direction $c$ for that path from that vertex.

Construct a graph $G=(V, E)$ as follows (See Fig. 55(a)). Add a node $v_{i}$ to $V$ for every $q_{i}$. Add an edge to $E$ between every pair of nodes $v_{i}, v_{j}$, with a weight of the minimum $M_{3}$ cost if the $\mathcal{C}$-directed paths from $q_{i}$ and $q_{j}$ enter $p$ from 
opposite directions. This allows each path to be matched in $G$, with the same cost as if they entered $p$ in opposite directions.

Next add a second node $u_{i}$ to $V$ for every $q_{i}$. Add an edge to $E$ between every pair of nodes $u_{i}, v_{i}$ with a weight of the minimum $M_{3}$ cost if the path from $q_{i}$ to $p$ is unmatched by a path entering $p$ in the opposite direction. Finally, add a zero weight edge between every node pair $u_{i}, u_{j}$.

Compute a minimum cost maximum cardinality matching $\eta$ on $G$. Transform back to the original problem by considering each pair of matched nodes in $\eta$. If both nodes correspond to the same child $q_{i}$, then the direction that gives the minimal $M_{3}$ cost independent of other children is taken for $q_{i}$ 's path to $p$. Otherwise, the nodes correspond to two different children in the original problem, and the their paths are made to enter $p$ in the pair of opposing directions that gave minimal $M_{3}$ costs.

Time complexity. The graph $G$ takes $\mathcal{O}\left(|\mathcal{C}| k^{2}\right)$ time to generate, and contains $\mathcal{O}(k)$ nodes and $\mathcal{O}\left(k^{2}\right)$ edges. Computing the matching therefore takes $\mathcal{O}\left(k^{3}\right)$ time. Hence, the total time complexity is $\mathcal{O}\left(\frac{r^{4}}{\varepsilon^{4}}|\mathcal{C}|^{3} n+\frac{r^{2}}{\varepsilon^{2}} \sum_{p \in \mathcal{S}}\left(|\mathcal{C}| d_{T}(p)^{3}+\right.\right.$ $\left.|\mathcal{C}|^{2} d_{T}(p)^{2}\right)$ ), where $d_{T}(p)$ is the degree of $p$ in $T$.

\subsection{Problem $\pi_{4}$}

In contrast to $\pi_{3}$, in $\pi_{4}$ we need only one path entering $p$ in a given direction to match many in the opposite direction.

Throughout this section, we say that a direction $c$ is used by a schematisation if there is a $\mathcal{C}$-directed path in the schematisation from at least one neighbour of $p$ that enters $p$ in direction $c$. The direction $c$ is not used if there is no such path.

Our approach again uses a transformation to a graph matching problem, this time matching edges to each of a subset $\mathcal{C}^{\prime} \subseteq \mathcal{C}$ of directions. The matching problem assumes that an optimal solution exists that uses every direction in the given subset $\mathcal{C}^{\prime}$, and does not use any other directions. To find a globally optimal solution, the algorithm processes all $2^{|\mathcal{C}|}$ subsets of $\mathcal{C}$ (assuming $k \geq|\mathcal{C}|$, otherwise only subsets of cardinality at most $k$ are considered). We expect $|\mathcal{C}|$ to be a small constant in most practical applications.

The algorithm. Process every subset $\mathcal{C}^{\prime} \subseteq \mathcal{C}$ for which $\left|C^{\prime}\right| \leq k$ as follows. Let $q_{1}, q_{2}, \ldots, q_{k}$ be the children of $p$ in $T$. For each $q_{i}, 1 \leq i \leq k$ and every direction $c \in \mathcal{C}^{\prime}$, calculate the $M_{4}$ cost if the $\mathcal{C}$-directed path from $q_{i}$ enters $p$ in direction $c$, with no path entering $p$ in the opposite direction. For now, store the solution that gives the lowest $M_{4}$ cost, breaking ties arbitrarily.

Once all children have been processed, an initial schematisation is constructed from the set of stored solutions. If this schematisation uses every direction $c \in \mathcal{C}^{\prime}$, then we are done; there is no possibility of reducing the end count, as an end will only be counted if there is an unused direction. If one or more directions are unused, we must satisfy our assumption of an optimal solution using exactly 
the set of directions $\mathcal{C}^{\prime}$, by choosing some of the paths to enter $p$ in the unused directions.

Compute for every direction $c \in \mathcal{C}^{\prime}$ the set of $\left|\mathcal{C}^{\prime}\right|$ children whose $\mathcal{C}$-directed paths to $p$ give the lowest additional $M_{4}$ cost if required to enter $p$ in direction $c$. Call this set $\operatorname{ch}_{c}(p)$. The additional $M_{4}$ cost for each child $q \in \operatorname{ch}_{c}(p)$ will be the cost difference between the initial schematisation and the one obtained if the path from $q$ enters $p$ in direction $c$.

Now construct a bipartite graph $G=(U, V, E)$ for matching (See Fig. 5 (b)). For each child $q_{i}$ that was added to $\operatorname{ch}_{c}(p)$ for any $c \in \mathcal{C}^{\prime}$, add exactly one node $u_{i}$ to $U$. For every direction $c_{j} \in \mathcal{C}^{\prime}$, add one node $v_{j}$ to $V$. Construct an edge between every pair of nodes $u_{i} \in U, v_{j} \in V$ for which it holds that $u_{i} \in \operatorname{ch}_{c_{j}}(p)$. Set the weight of the edge $u_{i}, v_{j}$ equal to the additional $M_{4}$ cost for the child $q_{i}$ 's $\mathcal{C}$-directed path to enter $p$ in direction $c_{j}$.

$G$ now contains $\mathcal{O}\left(\left|\mathcal{C}^{\prime}\right|^{2}\right)$ nodes and $\mathcal{O}\left(\left|\mathcal{C}^{\prime}\right|^{2}\right)$ edges. Compute a matching from $U$ to $V$ as in Section 4.3 . Once a matching is computed, modify the initial schematisation as follows. For each matched pair of nodes $u_{i} \in U, v_{j} \in V$, replace the initial path from $q_{i}$ to $p$ with a minimum-bend $\mathcal{C}$-directed path that enters $p$ in direction $v_{j}$. All other paths remain as in the initial schematisation. We now have a schematisation that uses every direction in the set $\mathcal{C}^{\prime}$, and is of minimal cost for $\mathcal{C}^{\prime}$.

After computing such a schematisation for all subsets $\mathcal{C}^{\prime} \subseteq \mathcal{C}$, take the schematisation that produces the minimum $M_{4}$ cost, breaking ties arbitrarily; this is an optimal solution. Note that if $k<|\mathcal{C}|$, the algorithm needs only to consider those subsets $\mathcal{C}^{\prime} \subseteq \mathcal{C}$ where $\left|\mathcal{C}^{\prime}\right| \leq k$.

Time complexity. The algorithm explores $\mathcal{O}\left(2^{|\mathcal{C}|}\right)$ subsets of directions. For each of these subsets, we can then populate the set $\operatorname{ch}_{c}(p)$ for every direction $c \in \mathcal{C}^{\prime}$ in $\mathcal{O}\left(|\mathcal{C}|^{2} k\right)$ time, since choosing the $i$ th largest of $n$ numbers can be done in $\mathcal{O}(n)$ time [1. The graph $G$ is constructed in $\mathcal{O}\left(|\mathcal{C}|^{2}\right)$ time, and $\mathcal{O}\left(|\mathcal{C}|^{4}|\log | \mathcal{C} \mid\right)$ time is needed to compute a matching from $G$. We therefore need $\mathcal{O}\left(\frac{r^{4}}{\varepsilon^{4}}|\mathcal{C}|^{3} k+\frac{r^{2}}{\varepsilon^{2}} 2^{|\mathcal{C}|}\left(|\mathcal{C}|^{6} \log |\mathcal{C}|+|\mathcal{C}|^{4} k\right)\right)$ time to process the vertex $p$, and $\mathcal{O}\left(\frac{r^{4}}{\varepsilon^{4}}|\mathcal{C}|^{3} n+\frac{r^{2}}{\varepsilon^{2}} 2^{|\mathcal{C}|}|\mathcal{C}|^{6} \log |\mathcal{C}| n\right)$ time for $T$.

\subsection{Problem $\pi_{5}$}

$\pi_{5}$ counts 1 end for each direction used if the opposing direction is not used, or 0 otherwise. Let $\mu\left(p^{\prime}, q^{\prime}, q, c_{1}, c_{j}\right)=M_{5}\left(q, q^{\prime}, c_{1}\right)+\delta\left(q^{\prime}, p^{\prime}, c_{1}, c_{j}\right)$ be the $M_{5}$ cost calculated by the algorithm of Section 4.1, before counting ends.

In order to count ends, we need only consider directions $c_{j} \in \mathcal{C}$ that correspond to a cost of at most 1 more than the minimum $\mu\left(p^{\prime}, q^{\prime}, q, c_{1}, c_{j}\right)$ value. By using two opposite directions for the $\mathcal{C}$-directed paths from two children $q_{1}, q_{2} \in \operatorname{ch}(p)$, we can save at most 2 from the $M_{5}$ cost. Hence, if either path entering $p$ in those directions had a $\mu\left(p^{\prime}, q^{\prime}, q, c_{1}, c_{j}\right)$ value of 2 or more greater than the optimal, there must be a pair of unopposed directions for the two paths with equal or lower $M_{5}$ cost. 
Given this fact, $\pi_{5}$ appears somewhat simpler than $\pi_{4}$. However, it can be shown to be NP-hard. Let CHILDPATHPlaCEMENT be the problem of choosing a direction $c_{j} \in \mathcal{C}$ for the path from each $q_{i} \in \operatorname{ch}(p)$ such that the corresponding schematisation gives a total $M_{5}$ cost of $m$.

\section{Theorem 1. ChildPathPlacement is NP-complete.}

The proof of Theorem 1 is by a straightforward reduction from SETCOVER; we omit it here due to space restrictions.

It is clear that ChildPAThPlacement can be solved if the $\pi_{5}$ problem can be solved, which leads to our final result for this section.

Corollary 1. $\pi_{5}$ is NP-hard.

$\pi_{5}$ can be solved using the same algorithm as $\pi_{4}$.

\section{References}

1. M. Blum, R. W. Floyd, V. R. Pratt, R. L. Rivest and R. E. Tarjan. Time bounds for selection. Journal of Comp. and Sys. Sciences, vol. 7, no. 4, p. 448-461, 1973.

2. U. Brandes, M. Eiglsperger, M. Kaufmann and D. Wagner. Sketch-driven orthogonal graph drawing. In Proc. Graph Drawing 2002, p. 1-11, 2002.

3. S. Cabello, M. de Berg and M. van Kreveld. Schematization of networks. Computational Geometry and Applications, vol. 30, p. 223-238, 2005.

4. S. Cabello and M. van Kreveld. Approximation algorithms for aligning points. Algorithmica, vol. 37, p. 211-232, 2003.

5. M. Eiglsperger, S. P. Fekete and G. W. Klau. Orthogonal graph drawing. Drawing Graphs, p. 121-171, Springer-Verlag Berlin Heidelberg, 2001.

6. H. N. Gabow. Data structures for weighted matching and nearest common ancestors with linking. In Proc. ACM-SIAM Symp. Discrete Alg., p. 434-443, 1990.

7. S.-H. Hong, D. Merrick and H. A. D. do Nascimento. The metro map layout problem. In Proc. Graph Drawing 2004, p. 482-491, 2004.

8. U. Lauther and A. Stübinger. Generating schematic cable plans using springembedder methods. In Proc. Graph Drawing 2001, p. 465-466, 2002.

9. L. Lovász and M. D. Plummer. Matching Theory. Elsevier, Amsterdam, 1986.

10. D. Merrick and J. Gudmundsson. Path simplification for metro map layout. Submitted to Graph Drawing, June 2006.

11. G. Neyer. Line simplification in restricted orientations. In Proc. of the 6th International workshop on Algorithm s and Data Structures, p. 13-24, 1999.

12. M. Nöllenburg and A. Wolff. A mixed-integer program for drawing high-quality metro maps. In Proc. Graph Drawing 2005, p. 321-333, 2006.

13. J. M. Stott and P. Rodgers. Metro map layout using multicriteria optimization. In Proc. Information Visualisation, p. 355-362, 2004. 